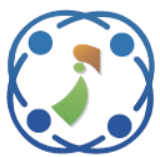

\title{
An Effective Method for Image Denoising Using Non-local Means and Statistics based Guided Filter in Nonsubsampled Contourlet Domain
}

\author{
Gollamandala Udaykiran Bhargava ${ }^{1 *}$ \\ Vaazi Gangadharan Sivakumar ${ }^{2}$ \\ ${ }^{I}$ Sathyabama Institute of Science and Technology, Chennai, India \\ ${ }^{2}$ Vidya Jyothi Institute of Technology, Hyderabad, Telangana, India \\ *Email: udaykiranbhargava25@gmail.com
}

\begin{abstract}
This article presents an effective method for image denoising using non-local means with packing of multi patches (NLM-PMP) in non-subsampled contourlet (NSC) domain which works based on multi-scale decomposition and directionality. Guided filter with image statistics (SGIF) is utilized to further process the obtained denoised image for mitigation of ringing artifacts those are invariably appeared, which leads to the local structure preservation like textures, edges and small details efficiently. The authors decomposed the image into low-pass subband coefficients and bandpass subband coefficients including the base and the detail scales. The low frequency noise in the base sub-band and the edges with small textural details in the detail scale are processed independently using an NLM-PMP filter. In addition, SGIF is applied to enhance the visual perception of denoised image. Experimental results indicate that the proposed approach is competitive at lower noise strength with respect to peak signal to noise ratio and structural similarity index measure and excels in performance at higher noise strength compared with several state-of-the-art algorithms.
\end{abstract}

Keywords: Image denoising, Non-local means, Guided filtering, Nonsubsampled contourlet transform, Peak signalto-noise ratio, Structural similarity index.

\section{Introduction}

The restoration of the original image from its noisy observation contaminated during acquisition, reception or transmission is a stepping stone for many image processing or computer vision tasks. Often the characteristics of noise depend on the sensor type, pixel dimension, exposure time, ISO speed, temperature, and ambient illumination level. However, in most of the applications, the distribution of noise can be characterised as additive white Gaussian noise (AWGN).

$$
y=z+\eta
$$

where $y$ is the observed (noisy) image, $z$ is the latent image and $\eta \in \aleph\left(0, \sigma^{2}\right)$ is the Gaussian noise of zero mean and $\sigma^{2}$ variance.

Available denoising methods can be broadly categorised into three classes: spatial, transform and dual domain approaches. The underlying idea behind spatial filtering techniques differs only to the extent of the weights (kernels) that are calculated either locally or non-locally to estimate different data points in an image [1]. Bilateral filter (BF) [2], non-local means filter (NLM) [3] and recently proposed guided image filter (GIF) [4] are few modern edge preserving filters that exploit either local, non-local or both self-similarity among the image patches for its restoration in the spatial domain. In contrast to spatial domain approaches, transform domain techniques represent signals with fewer non-zero coefficients. The energy-compaction property of several multi-resolution signal transformation $T$ is proved to be suitable for preserving the key signal components of the image by thresholding out the noise. The universal VisuShrink [5], the unbiased estimator-based SureShrink [6], the Bayesian prior-based BayesShrink [7], the statistical co-dependent 
bivariate shrinkage-based BiShrink [8], the probabilistic shrinkage function-based ProbShrink [9], the linear weight estimation-based Stein's unbiased risk estimate-linear expansion of thresholds (SURE-LET) [10] and the recently proposed NeighShrink [11] are few thresholding techniques that were applied in wavelet domain to preserve the high magnitude signal coefficients. However, the inability of wavelets in representing the curved edges $\left(\mathrm{C}_{2}\right.$ singularity $)$ degrades the performance of wavelet-based image denoising approaches. The implementation of anisotropic scaling in curvelet transform aides in representing image edges more sparsely compared with wavelets. Starck et al. [12] employed hard thresholding on the magnitude of complex curvelet coefficients for denoising. However, these transform domain-based thresholding (shrinkage) techniques suffer from inevitable ringing artefacts due to a sudden jump in coefficient magnitudes. Dual domain or hybrid approaches combine the advantages of both spatial and transform domain techniques to improve the overall image denoising quality. The multiresolution $\mathrm{BF}(\mathrm{MBF})$ incorporated both $\mathrm{BF}$ and wavelet thresholding in the approximation and the detail scales to suppress simultaneously the coarser grain (or low-frequency) and fine grain (or highfrequency) noise [13]. Knaus et al. [14] proposed a dual domain image denoising method by integrating joint $\mathrm{BF}$ and short time Fourier transform-based wavelet shrinkage technique. The inability of wavelets in representing edges [15] and the limitation of $\mathrm{BF}$ in preserving gradient direction of edges may degrade the overall performance of such techniques. Recently, NLM filter instead of BF has been considered in several kinds of literature to improve the performance of hybrid domain approaches [16] and [17]. Further, the procedure of denoising is considered as pre-processing stage in [18]. However, the block matching 3D collaborative filter (BM3D) excelled in denoising by grouping the similar (non-local) patches and collaboratively filtering the 3D blocks using $1 \mathrm{D}$ wavelet thresholding [19]. Though BM3D is considered the state-of-the-art technique, it is still unable to denoise few homogeneous regions that manifest as lowfrequency noise [20]. Moreover, the patch-based methods that search for a greater number of patches with similar local spatial structures may reduce at a higher noise level and thus restricts the performance of denoising. Motivated by the fact that the residual sparsity among the non-local similar patches can be reduced under a constrained prior model, recently Zha et al. [21] proposed a new image denoising technique - called group sparsity residual constrain
(GSRC) - to enhance the performance of group sparse-based methods (that includes state-of-the-art BM3D technique). Though the GSRC technique achieves numerically improved results with a modest increase in visual quality at higher noise strength, the iterative method puts higher computational burden. Similarly, the sophisticated Cauchy filter-based image denoising technique provides comparable results with higher computation complexity [22]. The NLM, an averaging filter utilises the similarity among the patches to denoise the image. Though it is very efficient in preserving edges, it is unable to process efficiently near the textured regions [23]. The energy compaction property of the curvelet that represents any signal in several scales can be used to separate various spatial frequencies of image efficiently. Recently, author in [24] presented a denoising approach that utilized the combination of nonsubsampled contourlet and bandelet transforms for efficient denoising performance. However, this approach ignores the artifacts those are introduced after the denoising procedure.

In [25], author incorporated the advantages of both (multiscale) NLM filtering and hard thresholding in three different scales of the curvelet: the approximation, the coarser and the fine scale. The edge preserving the property of the non-linear NLM filter ensures the suppression of noise in the approximation scale and aids in preserving well connected edges with small image details in the fine scale. Unlike multiscale filtering, the hard thresholding in the coarser scale at different orientations is employed to separate the signal from the insignificant noise coefficients. The inevitable ringing artefacts in the reconstructed image are further processed by GIF to obtain the final denoised image. The results of this on both grayscale and colour images have shown encouraging quantitative and qualitative improvement compared with several state-of-the-art techniques at higher noise strength. However, this technique unable to preserve the finescale details at lower noise strength and it is quite hard to sampling on a grid of rectangular for the transform presented in [25] since it was implemented in continuous and the directions apart from horizontal and vertical are very unlike on the grid of rectangular.

To address this issue, an effective method for image denoising using non-local means with packing of multi patches (NLM-PMP) in nonsubsampled contourlet (NSC) domain is proposed, which works based on multi-scale decomposition and directionality. Guided filter with image statistics (SGIF) is utilized to further process the obtained 
denoised image for mitigation of ringing artifacts those are invariably appeared, which leads to the local structure preservation like textures, edges and small details efficiently. The performance of the proposed hybrid technique is tested on greyscale images and it works well at low-noise strengths. The experimental results compared with several state-ofthe-art techniques demonstrate the competitiveness of the proposed approach at lower noise strength, while yielding better performance at a lower value of $\sigma$.

The rest of the article is organised as follows: we first explained the procedures of NLM, NSCT and SGIF in Section 2. The proposed algorithm is explained in Section 3. The experimental results in terms of denoising quality and computational complexity are presented and discussed in Section 4. Finally, Section 5 concludes the paper.

\section{Background: NLM, NSCT and SGIF}

\subsection{Non-local means}

The basic principle of NL-means is simple and intuitive, in which weighted average is utilized to estimate the true value of clean pixel by exploring like pixels, where exponential decomposition of weights occurs with the mitigation of similarities. More precisely, let $U_{i}$ and $V_{i}$ be the original pixel value and the added noise, for $i=1,2, \ldots, M$, where $M$ is the number of pixels in the image and hence the observed noisy pixel is $U_{i}+V_{i}=Z_{i}$. NLmeans estimates $U_{i}$ as

$$
\widehat{Z_{\iota}}=\frac{1}{C} \sum_{j \in N_{i}} W_{i j} Z_{j}
$$

Where $N_{i}$ denotes the search window centered at $\mathrm{i}$, which could be as large as the whole image and usually chosen empirically, $\left\{W_{i j} \mid j \in N_{i}\right\}$ are the weights.The weight $W_{i j}$ is determined as an emphasizing function of the resemblance or parallelly a mitigating function in some distance $U_{i}$ and $U_{j}$, which are usually strange and $Z_{i}$ and $Z_{j}$ are utilized instead. NLM follows well known and most effective distance measurement named squared Euclidean distance. More exactly, the formula for the distance between two pixels those are noisy is

$$
D_{\text {pixel }}\left(Z_{i}, Z_{j}\right) \stackrel{\text { def }}{=}\left(Z_{i}-Z_{j}\right)^{2}-2 \sigma^{2}
$$

NLM expands to the comparison of pixel to the comparison of patch to induce the distance stronger to noise. Similar patches have similar centres based on the observation that in natural images and utilized the following squared distance i.e., based on patch

$$
D_{\text {patch }}\left(Z_{i}, Z_{j}\right) \stackrel{\text { def }}{=} \sum_{k=1}^{d}\left(Z_{i}(k)-Z_{j}(k)\right)^{2}-2 d \sigma^{2}
$$

Where $Z_{i}(k)$ and $Z_{j}(k)$ are the pixels in the patches centered at the $i_{t h}$ and $j_{t h}$ pixels respectively, and $\mathrm{d}$ is the number of pixels in patch. NL-means uses an exponential kernel as the weight function:

$$
W_{i j} \stackrel{\text { def }}{=} \exp \left(-\frac{\max \left\{D_{\text {patch }}\left(Z_{i}, Z_{j}\right), 0\right\}}{d \sigma^{2} T^{2}}\right)
$$

Where $d \sigma^{2}$ is for normalization, $T$ is a decay parameter and max are used so that the weight is set to 1 when the distance is negative. The above process denoises the image pixel by pixel. This has been extended to patch wise implementation. Similar to the pixel wise process, a weight function is defined between two patches, but each patch is denoised as a weighted average of all patches centered in the search window of the first patch.

\subsection{Non-subsampled contourlet transform}

Contourlet transform (CT) is an approach of two-dimensional transform [26] which is utilized to represent an image with several properties like multiresolution, anisotropy, localization, critical sampling and directionality. Multi-scaling and directionality are the basis of CT. Ideally, few coefficients extracted from the image by utilizing CT can represent the image contours effectively. Human visual system (HVS) and curvelet transform are the motivated concepts of CT. The curvelet transform [27], which can grasp the smoothness of image contours with unlike stretched shapes and in mixture of directions. However, the curvelet transform was implemented in continuous domain only and the directionality will be different on rectangular grid. Hence, CT is developed in discrete domain with multiresolution and multi directionality. CT is an integration of Laplacian pyramid (LP) and directional filter banks (DFB) which are double filter bank structures and the same also called as a pyramid directional filter bank (PDFB). Only one band-pass image will be obtained by the utilization of LP decomposition, which avoids scrambling of frequency. DFB suits for higher frequencies only since it leaks the low frequency at directional sub bands. 


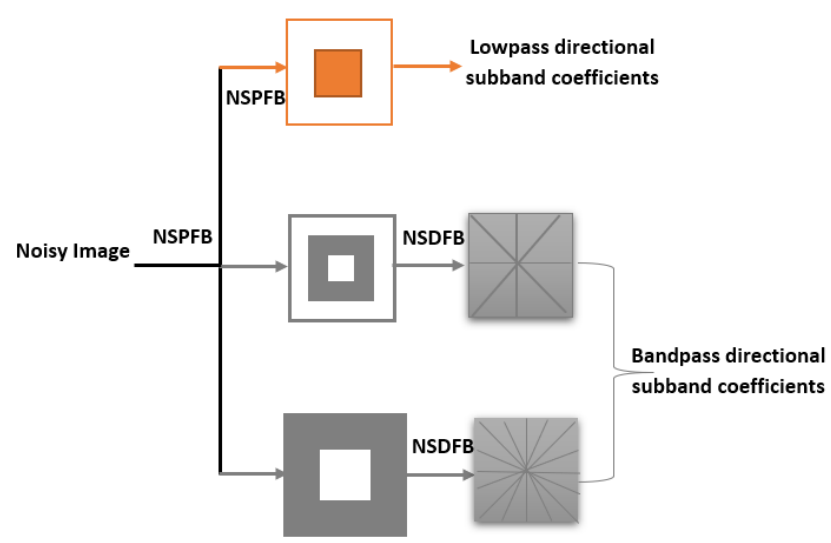

Figure. 1 Structure of NSCT frame work with the integration of NSPFB and NSDFB

However, the CT is not without flaws since it is not a shift invariant due to the up and down samplings presence in the filter bank structures. To regain the $\mathrm{CT}$ multiscale and directional properties, non-subsampled filter bank (NSFB) versions of LP and DFB structures is replaced with the conventional filter banks. Fig. 1 demonstrates that the construction of integration of the NSPFB and NSDFB to implement NSCT [28]. Care must be taken in NSCT construction when DFB is applied to the coarser scales of the LP. Due to the treestructure nature of NSDFB, the response of directionality at the frequencies of lower and upper suffers from the effect of aliasing, which can be a serious issue in later pyramid stages, where the DFB pass band region is labelled as "Good" or "Bad." Hence, one can view that for coarser scales, the high-pass channel in effect is filtered with the DFB pass band bad portion, which leads to the effect of severe aliasing and in some observed cases a considerable loss of directional resolution. This problem gets overcome by judiciously up sampling the NSDFB filters.

\subsection{Statistics based guided image filter (SGIF)}

The proposed SGIF method is explained as follows:

If $\mathcal{G}$ is a guided image centered at a pixel $m$ in a local square window $w$, then the filtered output (0) at a pixel $n$ is given by

$$
\mathbb{O}_{n}=a_{m} \mathcal{G}_{n}+b_{m}, \forall n \in w_{m}
$$

Where $a_{m}$ and $b_{m}$ are the linear coefficients which are constant in window $w_{m}$. To determine linear coefficients, constraints have to be derived from the input image II. In other way, to get noise free output, unwanted components $\mathbb{N}$ (like noise or texture) must be subtracted from $\mathbb{I}$.

$$
\mathbb{O}_{n}=\mathbb{I}_{n}-\mathbb{N}_{n}
$$

The solution for this problem should minimize the difference between $\mathbb{I}$ and $\mathbb{O}$. It should also maintain the relation in Eq. (7). Hence, $a_{m}$ and $b_{m}$ are the linear coefficients that can minimize the cost function in window $w_{m}$ as

$$
\begin{aligned}
& E\left(a_{m}, b_{m}\right)=\sum_{n \in w_{m}}\left\{\left(a_{m} \mathcal{G}_{n}+b_{m}-\mathbb{I}_{n}\right)^{2}+\right. \\
& \left.r a_{m}{ }^{2}\right\}
\end{aligned}
$$

where $r$ is the regulization parameter. Eq. (8) represents the linear regression model. The solution for this is directly given by

$$
\begin{aligned}
& a_{m}=\frac{\frac{1}{|w|} \sum_{n \in w_{m}} \mathbb{S}_{n} \mathbb{I}_{n}-\mu_{m} \overline{\mathbb{I}}_{n}}{\sigma_{m}^{2}+r} \\
& b_{m}=\overline{\mathbb{I}}_{n}-a_{m} \mu_{m}
\end{aligned}
$$

Here $|w|$ is the number of pixels in a window $w_{m}$ centered at pixel $m, \mu_{m}$ is the mean, and $\sigma^{2}{ }_{m}$ is the variance in the window $w_{m} \cdot \overline{\mathbb{I}}_{n}$ is the mean of input $\mathbb{I}_{n}$ in $w_{m}$ and is given by

$$
\overline{\mathbb{I}}_{n}=\frac{1}{w} \sum_{n \in w_{m}} \mathbb{I}_{n}
$$

Once linear coefficients are obtained, then output $\mathbb{O}_{n}$ can be solved according to Eq. (6). But different overlapping windows $w_{m}$ centered at $m$ contain pixel $n$ in common. To resolve this problem, take average of all estimates of $\mathbb{O}_{n}$. Hence, the filtering output can be given as

$$
\mathbb{O}_{n}=\overline{a_{m}} \mathcal{G}_{n}+\overline{b_{m}}
$$

Where $\quad \overline{a_{m}}=\frac{1}{w} \sum_{n \in w_{m}} a_{m} \quad$ and $\quad \overline{b_{m}}=$ $\frac{1}{w} \sum_{n \in w_{m}} b_{m}$ are the averages of all linear coefficients. In this article, filtering output of guided image II in the guiding of $\mathcal{G}$ is denoted as $\operatorname{SGIF}_{\mathfrak{y}, r}(\mathbb{I}, \mathcal{G})$, where $\mathfrak{y}$ is the filter size/neighborhood size and $r$ is the degree of smoothing/regulization parameter. The behaviour of the SGIF controlled by these parameters $\mathfrak{y}$ and $r$. If the guided image has a variance $\sigma^{2}$ higher than the threshold $r\left(\sigma_{m}^{2} \geq r\right)$, within a window $w_{m}$, then the pixel in the center of the window remain unchanged, whereas if a pixel is in the centre of low variance window whose variance is less than, then pixel value is replaced by the average of the neighbourhood.

The basic idea is to find weight corresponding to 


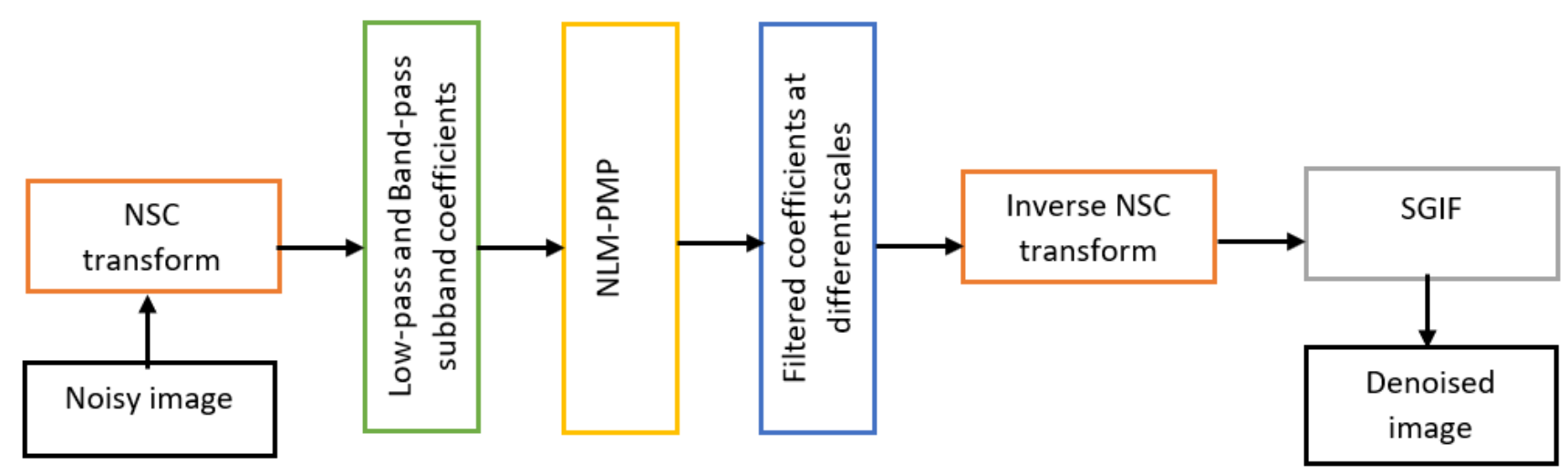

Figure. 2 Proposed image denoising framework

a pixel in an image based on its horizontal and vertical edge strengths. In theory, to find a weight corresponding to a pixel at a location $(m, n)$ in an image take a square window $w$ of size $p \times p$ around its neighbourhood. Consider $\mathbb{Q}$ as a matrix and find its covariance matrix by considering row as an observation, column as a variable.

$$
\operatorname{cov}(\mathbb{Q})=E\left[(\mathbb{Q}-E[\mathbb{Q}])(\mathbb{Q}-E[\mathbb{Q}])^{T}\right]
$$

Calculate unbiased horizontal estimate of a covariance matrix at a pixel location $(m, n)$ as

$$
\mathfrak{U}_{\mathcal{E}_{\mathrm{H}}}^{m, n}(\mathbb{Q})=\frac{1}{p-1} \sum_{h=1}^{p}\left(\mathbb{Q}_{k}-\overline{\mathbb{Q}}\right)\left(\mathbb{Q}_{k}-\overline{\mathbb{Q}}\right)^{T}
$$

Where $\mathbb{Q}_{k}$ is the $k^{\text {th }}$ observation of the $p$ dimensional variable and $\overline{\mathbb{Q}}$ is the average of the observation. Interestingly diagonal of $\mathfrak{U}_{\mathcal{E}_{\mathrm{H}}^{m}}^{m, n}(\mathbb{Q})$ is a variance vector. Compute Eigen values $\lambda_{\varepsilon_{\mathrm{H}}^{k}}^{k}$ of $\mathfrak{U}_{\mathcal{E}_{\mathrm{H}}}^{m, n}(\mathbb{Q})$. As the size of matrix is $p \times p$, number of Eigen values can be found is $p$. To get horizontal edge strength $e_{\varepsilon_{\mathrm{H}}}$, add all these Eigen values.

$$
\mathrm{e}_{\mathcal{E}_{\mathrm{H}}}(m, n)=\sum_{k=1}^{p} \lambda_{\mathcal{E}_{\mathrm{H}}}^{h}
$$

Similarly, to take vertical edge strength into account, take every column as an observation and row as a variable. Calculate the unbiased vertical estimate $\mathfrak{H}_{\mathcal{E}_{v}}^{m, n}$, and then compute the Eigen values $\lambda_{\mathcal{E}_{v}}^{k}$. Add these Eigen values to get the vertical edge strength $e_{\varepsilon_{v}}$ as,

$$
\mathrm{e}_{\mathcal{E}_{\mathcal{V}}}(m, n)=\sum_{k=1}^{p} \lambda_{\mathcal{E}_{\mathcal{V}}}^{k}
$$

To find the weight $\mathbb{W}(m, n)$ of a pixel at location $(m, n)$, take a sum of $\mathbf{e}_{\mathcal{E}_{\mathrm{H}}}(m, n)$ and $\mathrm{e}_{\varepsilon_{v}}(m, n)$

$$
\mathbb{W}(m, n)=\mathbf{e}_{\varepsilon_{\mathrm{H}}}(m, n)+\mathbf{e}_{\mathcal{E}_{\mathcal{V}}}(m, n)
$$

\section{Proposed frame work}

Image denoising using non-local means with packing of multi patches (NLM-PMP) in nonsubsampled contourlet (NSC) domain is proposed. In addition, statistics based guided image filter (SGIF) is utilized to enhance the denoised image visual quality by mitigating the ringing artifacts. Fig. 2 demonstrates that the proposed image denoising frame work. NSCT utilized for obtaining the multiscale decomposition and multi directional coefficients which provides the spatial and spectral information at different scales and different directions as well. This motivates the authors to utilize both the NSCT and NLM-PMP for getting the enhanced denoising performance even at higher noise levels with improved qualitative performance both in terms of quality assessment and visual perception with enhanced textured regions. Further, statistics based guided image filter (SGIF) is utilized for better preservation of local structures like edges, textures and small details.

\footnotetext{
Algorithm 1: Proposed hybrid image denoising

Step 1: Select and read a noisy image

Step 2: Apply NSC transform to decompose the noisy image using non-subsampled Laplacian pyramid filter banks for multi scale decomposition and non-subsampled directional filter bank for multi dimensionality (refer section 2.2).
}

Step 3: Obtain the low-pass subband and band-pass subband directional coefficients from the NSCT frame work.

Step 4: Filter the coefficients of NSCT using NLMPMP methodology (refer section 3.1)

Step 5: Obtain filtered coefficients at different scale regions using NSCT and NLM-PMP approaches.

Step 6: Apply inverse NSC transform to obtain the 
spatial filtered output.

Step 7: Compute the SGIF to get retain the textured regions at higher noise levels for the obtained output at step 6.

\subsection{Non-local means with packing of multiple patches (NLM-PMP)}

The NLM-PMP is based on the idea of separable filtering which is common in the image processing literature. For example, in [29], author presented bilateral filtering based separable formulations and similarly NLM based separable formulation was presented in [30]. These filters are not separable since they can't execute the process of filtering by fast processing of columns and rows (as can be done for linear filters with separable kernels). As a matter of fact, filtering results of row adopted by column result is ideally dissent from the results obtained by the operations done in reversible manner. Therefore, in our proposal authors considered both the possibilities into account and derived the denoised output image as these primitive's optimization. The optimality is in the sense of a certain replacement of the mean-squared error that is popularly referred to as Stein's unbiased risk estimator [31], [32] which resolves the $2 \times 2$ linear system. The methodology presented in this is differ from that the separable bilateral algorithm and gives much better results of denoising framework.

The major novelty of NLM-PMP, however, is the proposal of an algorithm that can reduce the complexity of one-dimensional NLM from $O(N S K)$ to $O(N S)$, where $\mathrm{N}$ is the length of the signal. This is established on the reflection that the distances of patch regarded in the NLM of a one-dimensional signal can be calculated from $O(N S)$ entries of a matrix which is specially implanted, which is found by applying a box or Gaussian filter along the matrix sub diagonals which is computed through lifting, namely, via the tensor product of the signal with itself. As is well-known, box and Gaussian filtering can be performed using $O(1)$ operations with respect to the filter length.

The NLM-PMP algorithm as an outcome need $\mathrm{O}(\mathrm{NS})$ operations to calculate the full patch set distances in NLM. To the best of author knowledge, the observation that lifting can be utilized for efficiently calculating the distances of patch is novel. We further utilize this lifting-based algorithm to implement a fast-separable NLM formulation for the images of 2D i.e., grayscale images. The proposed NLM-PMP complexity is $2 N \times O(N S)=O\left(N^{2} S\right)$ for an $N \times N$ image, which is substantially smaller than the $O\left(N^{2} S^{2} K^{2}\right)$ complexity of standard NLM.

\section{Results and discussion}

This section describes the experimental analysis of proposed denoising system. The denoising quality of the proposed algorithm is evaluated qualitatively as well as quantitatively for grayscale images. The experiments were conducted on a few standard grayscale images as shown in Fig. 3. To perpetuate uniformity in comparison, all the images were contaminated with simulated gaussian noise of standard deviations, $\sigma=[10,20,30,40]$.

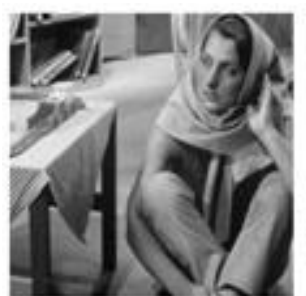

(a)

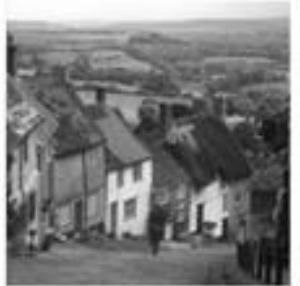

(f)

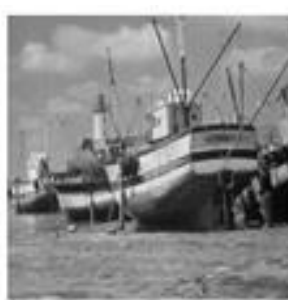

(b)

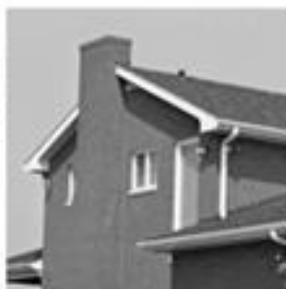

(g)

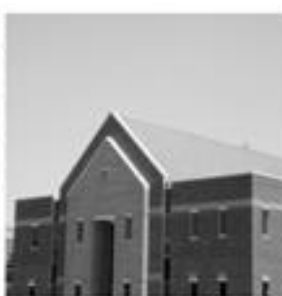

(c)

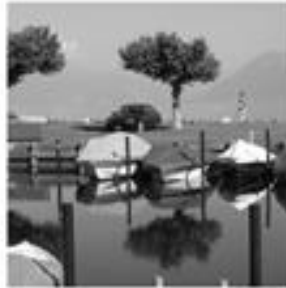

(h)

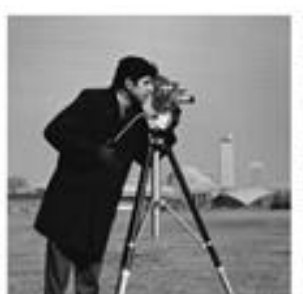

(d)

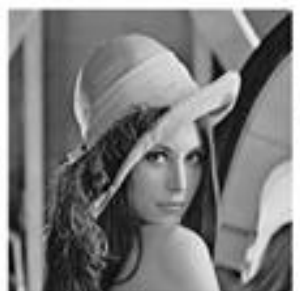

(i)

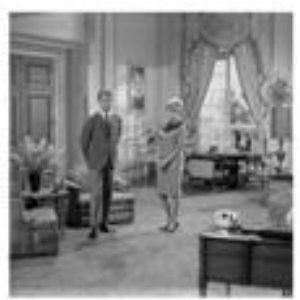

(e)

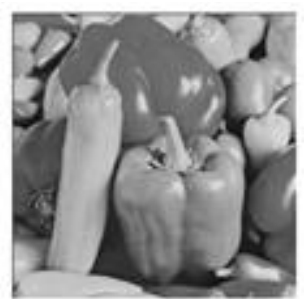

(j)

Figure. 3 Test images of: (a) Barbara, (b) Boat, (c) Building, (d) Cameraman, (e) Couple, (f) Goldhill, (g) House, (h) Lake, (i) Lena, and (j) Peppers 


\subsection{Quality evaluation parameters}

Quality assessment of images is a vital part in any application. It gives the mathematical proof of specific methodologies robustness, imperceptibility and efficiency. Here we considered few parameters to prove the algorithm stability and efficiency over BW applications. The quality metrics utilized here are like peak signal-to-noise ratio (PSNR), mean square error (MSE) and structural similarity (SSIM) index. PSNR can be formulated as,

$$
\begin{gathered}
P S N R=10 \log _{10} \frac{255^{2}}{M S E} \\
M S E=\frac{1}{M \times N} \sum_{x=0}^{M-1} \sum_{y=0}^{N-1}[I(x, y)-O(x, y)]^{2}
\end{gathered}
$$

Where $M$ and $N$ are the number of rows and number of columns in an image, $x$ and $y$ represents the spatial coordinates, $I$ denote original image and $O$ is a denoised image.

The SSIM index is calculated as

$$
\operatorname{SSIM}(x, y)=\frac{\left(2 \mu_{x} \mu_{y}+c_{1}\right)\left(2 \sigma_{x y}+c_{2}\right)}{\left(\mu_{x}^{2}+\mu_{y}^{2}+c_{1}\right)\left(\sigma_{x}^{2}+\sigma_{y}^{2}+c_{2}\right)}
$$

Where

$\mu_{x}$ and $\mu_{y}$ are the mean of $\mathrm{x}$ and $\mathrm{y}$.

$\sigma_{x}{ }^{2}$ and $\sigma_{y}{ }^{2}$ are the variances of $\mathrm{x}$ and $\mathrm{y}$.

$\sigma_{x y}$ is the covariance between $\mathrm{x}$ and $\mathrm{y}$.

$c_{1}=\left(k_{1} L\right)^{2}$ and $c_{2}=\left(k_{2} L\right)^{2}$ are two variables to stabilize the division with weak denominator.

$L$ is the dynamic range of the pixel values, $k_{1}=$ 0.01 and $k_{2}=0.03$

\subsection{Visual analysis}

The denoising quality of the proposed algorithm was evaluated and compared using PSNR (in dB) and SSIM indices. CT [12], NLM filtering [3], MBF [13], NLM method noise thresholding (NLMNT) [17] and the state-of-the-art BM3D [19], GSRC [21] and CT-NLM-GF [25] are considered in denoising quality comparison for the grayscale image. Fig. 4 disclose that the denoised outcome of the proposed and conventional denoising approaches with a value of $\sigma=10$. It is clear that the obtained denoised image of proposed model looks more qualitative over the other denoising models where the texture information is lost. Similarly, for the values of $\sigma=$ 20,30 and 40, the obtained denoising results are shown in Fig. 5, Fig. 6 and Fig. 7 respectively.

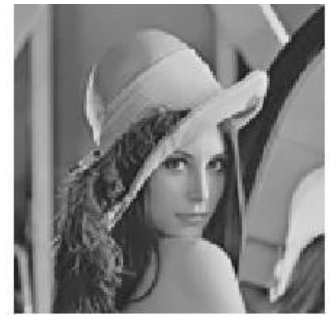

(c)

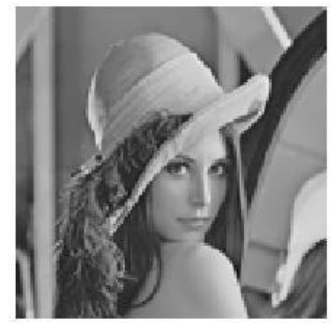

(g)

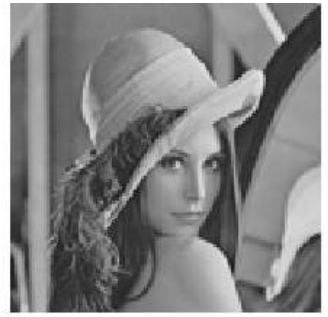

(d)

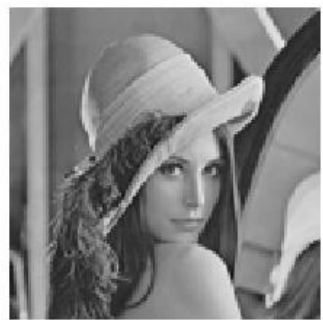

(h)

(e)

(f)

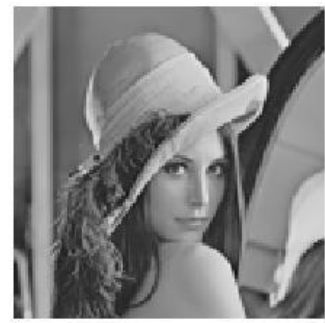

Figure. 4 Denoised output images of 'Lena' using the: (a) CT [12], (b) NLM [3], (c) MBF [13], (d) NLMNT [17], (e) BM3D [19], (f) GSRC [21], (g) CT-NLM-GF [25], and (h) proposed denoising model with $\sigma=10$ 


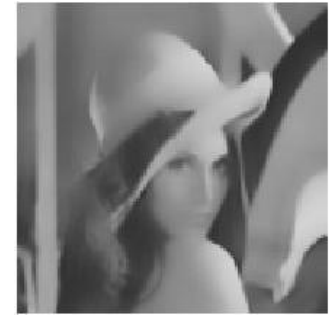

(a)

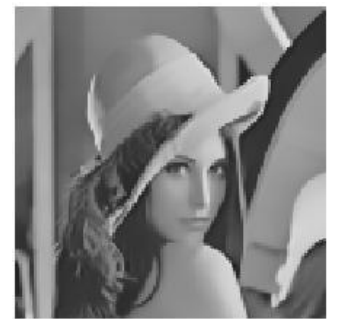

(e)

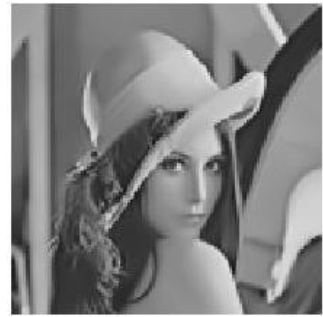

(b)

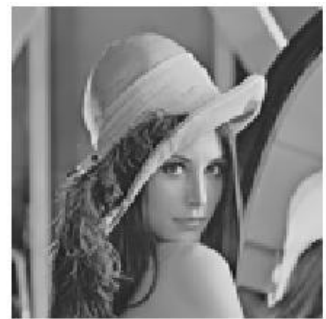

(f)

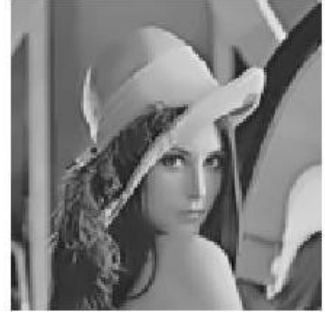

(c)

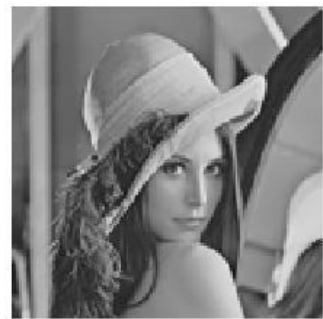

(g)

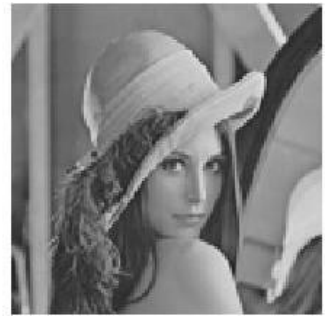

(d)

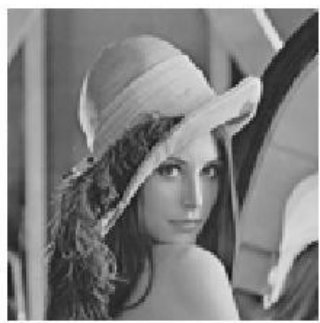

(h)

Figure. 5 Denoised output images of 'Lena' using the: (a) CT [12], (b) NLM [3], (c) MBF [13], (d) NLMNT [17], (e) BM3D [19], (f) GSRC [21], (g) CT-NLM-GF [25], and (h) proposed denoising model with $\sigma=20$

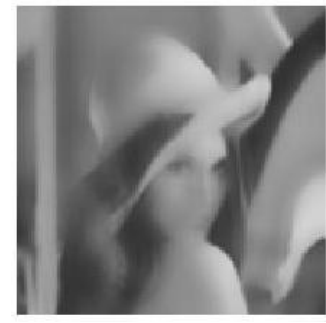

(a)

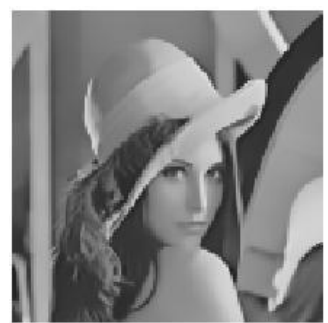

(e)

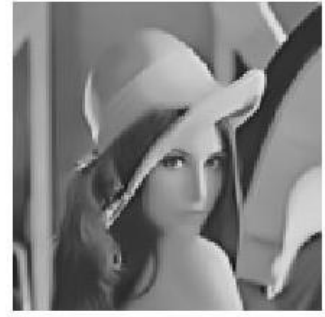

(b)

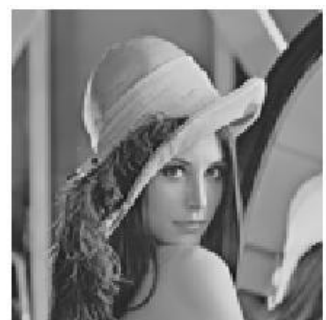

(f)

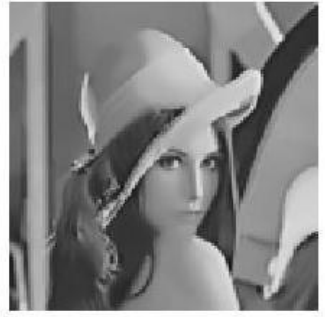

(c)

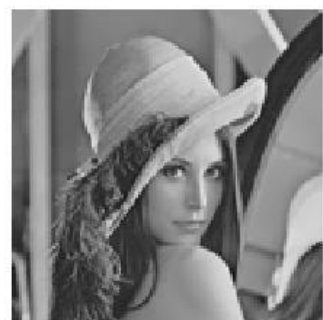

(g)

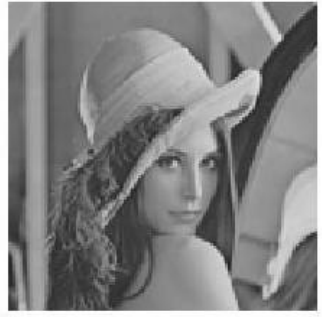

(d)

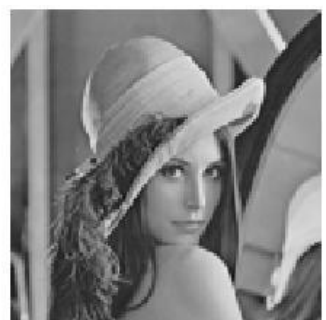

(h)

Figure. 6 Denoised output images of 'Lena' using the: (a) CT [12], (b) NLM [3], (c) MBF [13], (d) NLMNT [17], (e) BM3D [19], (f) GSRC [21], (g) CT-NLM-GF [25], and (h) proposed denoising model with $\sigma=30$ 


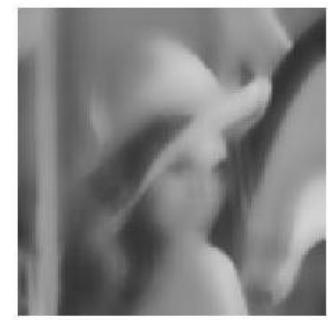

(a)

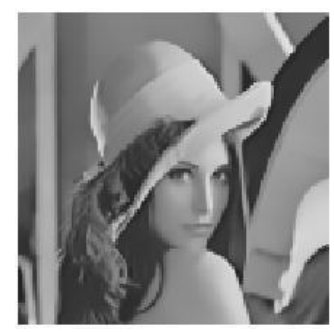

(e)

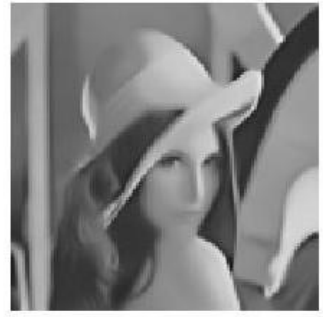

(b)

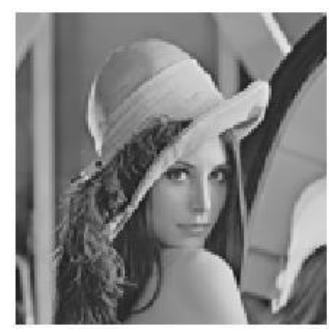

(f)

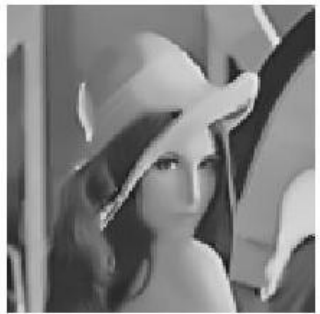

(c)

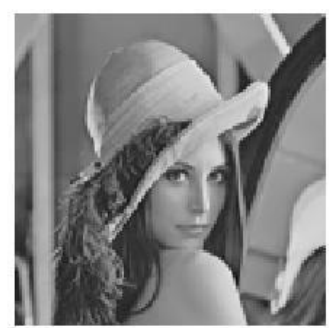

(g)

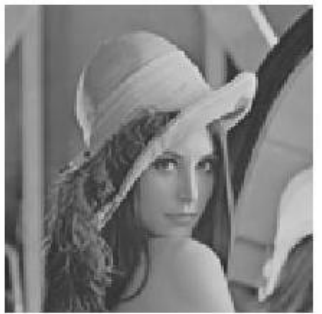

(d)

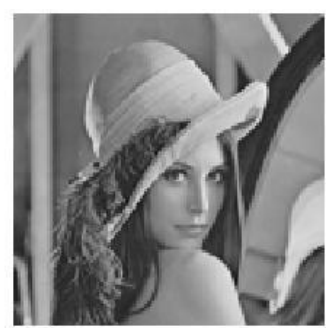

(h)

Figure. 7 Denoised output images of 'Lena' using the: (a) CT [12], (b) NLM [3], (c) MBF [13], (d) NLMNT [17], (e) BM3D [19], (f) GSRC [21], (g) CT-NLM-GF [25], and (h) proposed denoising model with $\sigma=40$

Table 1. Mean PSNR (dB) and SSIM measure between original and denoised images for different denoising approaches on test images in Fig. 3

\begin{tabular}{ccccccccc}
\hline & \multicolumn{4}{c}{ PSNR in dB } & \multicolumn{4}{c}{ SSIM } \\
Denoised methods & $\sigma=10$ & $\sigma=20$ & $\sigma=30$ & $\sigma=40$ & $\sigma=10$ & $\sigma=20$ & $\sigma=30$ & $\sigma=40$ \\
\hline CT [12] & 32.592 & 29.594 & 28.043 & 26.96 & 0.938 & 0.88 & 0.834 & 0.797 \\
NLM [3] & 35.10 & 31.452 & 29.035 & 27.252 & 0.956 & 0.901 & 0.839 & 0.776 \\
MBF [13] & 33.38 & 30.107 & 28.32 & 27.14 & 0.934 & 0.882 & 0.841 & 0.805 \\
NLMNT [17] & 34.74 & 31.69 & 29.597 & 27.91 & 0.958 & 0.907 & 0.853 & 0.798 \\
BM3D [19] & 36.15 & 32.70 & 30.57 & 28.766 & 0.965 & 0.928 & 0.889 & 0.850 \\
GSRC [21] & 35.37 & 32.32 & 30.652 & 29.02 & 0.958 & 0.918 & 0.887 & 0.858 \\
CT-NLM-GF [25] & 35.44 & 32.08 & 30.52 & 29.08 & 0.962 & 0.919 & 0.896 & 0.864 \\
Proposed & $\mathbf{4 3 . 6 3}$ & $\mathbf{3 5 . 0 7}$ & $\mathbf{3 1 . 8 2}$ & $\mathbf{2 9 . 3 8}$ & $\mathbf{0 . 9 9 8}$ & $\mathbf{0 . 9 9 3 3}$ & $\mathbf{0 . 9 8 6}$ & $\mathbf{0 . 9 7 5 6}$ \\
\hline
\end{tabular}

\subsection{Quantitative analysis}

Table 1 demonstrates that the quality evaluation parameters like PSNR and SSIM for the denoising algorithms like CT [12], NLM [3], MBF [13], NLMNT [17], BM3D [19], GSRC [21], CT-NLMGF [25] and proposed denoising model with the $\sigma$ values ranging from 10 to 40, where our proposed model obtained the best values of both PSNR and SSIM which are highlighted in bold letter for visual convenience. However, here mean PSNR is considered instead of individual image PSNR which is further compared with conventional denoising approaches as demonstrated in Table 1.

The comparative quality analysis of proposed and conventional denoising approaches with PSNR and SSIM is disclosed in Fig. 8 and Fig. 9 respectively.

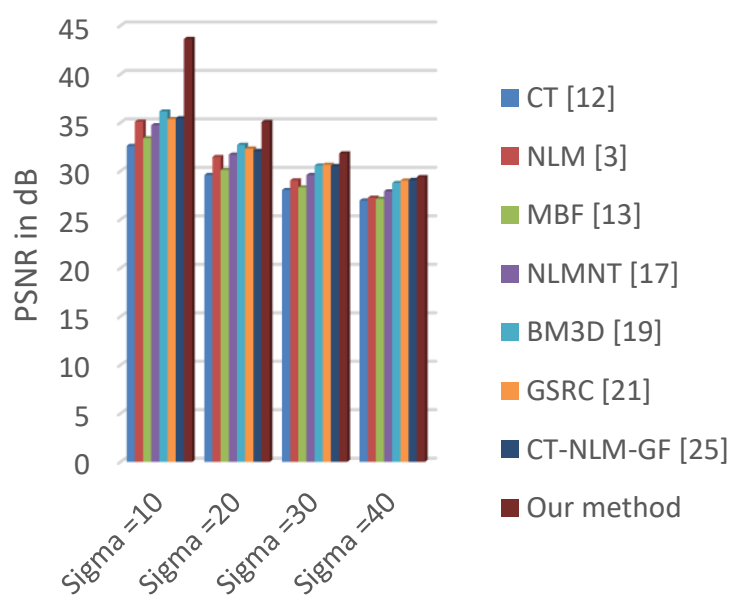

Figure. 8 Performance comparison of proposed and conventional denoising algorithms with PSNR for various values of $\sigma$ 


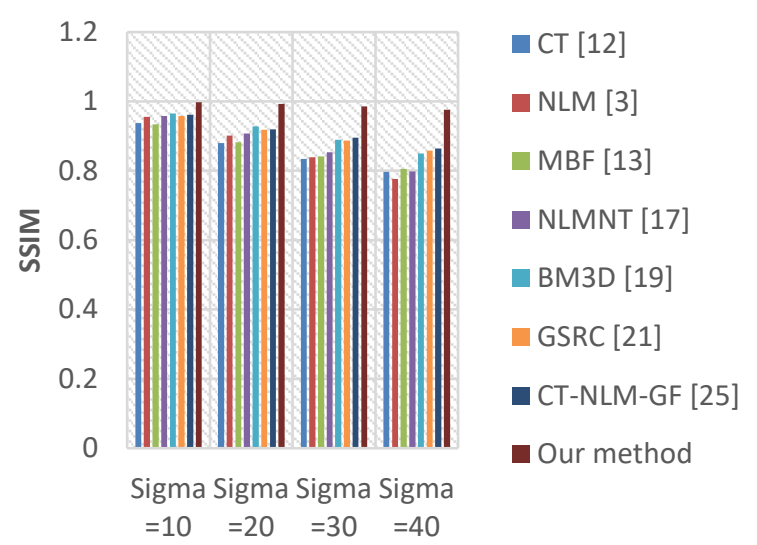

Figure. 9 SSIM values of proposed and existing denoising models with different $\sigma$ values

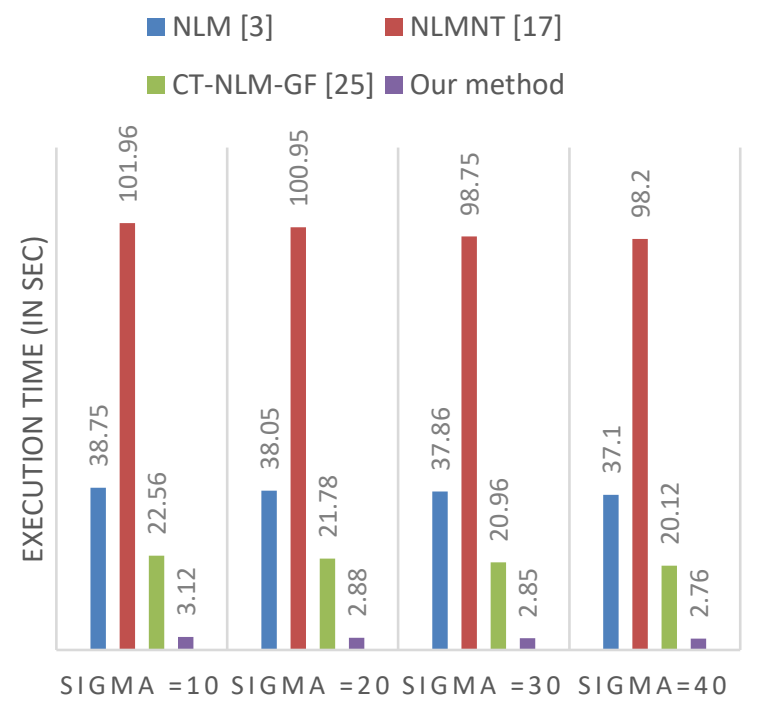

Figure. 10 Comparison of execution time (in sec) for the proposed and existing denoising approaches with an image of size $512 \times 512$

\subsection{Computational complexity}

Further, the computational complexity also considered to disclose the efficacy and robustness of proposed hybrid denoising framework with comparison to the other denoising algorithms discussed in the literature. Table 2 demonstrates that the execution time (in sec) of the denoising algorithms with the results disclosed in Figs. 4-7.

The utilization of NLM-PMP and SGIF algorithms in our NSC-based hybrid denoising approach assists in understating the complexity denoising of a digital image complexity. In addition, the operation speed of proposed model is enhanced by restricting the level of decompositions. Like the quality evaluation parameter comparison which is done for the proposed and conventional denoising

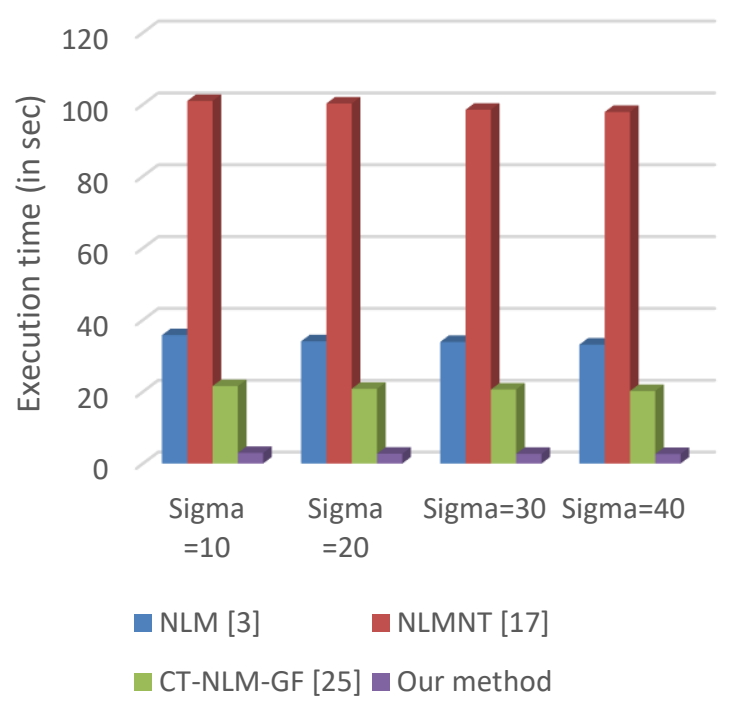

Figure. 11 Performance of execution time (in sec) with the proposed and conventional denoising schemes for an image with size $256 \times 256$

approaches disclosed in Table 1, the execution time complexity also done with the same approaches as NLM [3], NLMNT [17] and CT-NLM-GF [25]. Computation of execution time is done on a RAM of size 4.00 GB, 64-bit Intel(R) Core ${ }^{\mathrm{TM}}$, i3，5005U CPU @ $2.00 \mathrm{GHz}$ PC using MATLAB R2016b environment.

Fig. 10 shows that the performance of time complexity with the image of size $512 \times 512$ for the denoising algorithms like NLM [3], NLMNT [17], CT-NLM-GF [25] and proposed hybrid model with variable $\sigma$. The existing denoising models consumed more time while executing the procedure of image denoising as shown in Fig. 10, where in our method the time complexity is reduced dramatically. In addition, an image with size $256 \times 256$ also tested for the time complexity analysis which is disclosed in Fig. 11.

\section{Conclusions}

Most image denoising methods assume that the signal is smooth or piecewise smooth and the noise is oscillatory. However, many fine structures and small details of an image are as oscillatory as noise, which poses challenges in image modelling. The proposed method analysed the noisy image in NSC domain to denoise both the smooth (low-frequency) and oscillatory (high-frequency) noise. Unlike the development of spatial domain, implementation of NLM-PMP filter in NSC domain extracts more adequately the self-similarity among the high correlated coefficients within individual scales with less computational time. In addition, the inevitable 
ringing artefacts in the reconstructed image (which is mostly ignored in many transform domain techniques) is further processed using SGIF to keep the sharp image edges with a minimum loss of fine details. Comparative analysis also given for several denoising approaches presented in the literature with respect to the quality metrics like PSNR and SSIM. Further, the computational complexity also calculated for showing the robustness of proposed denoising method with other approaches.

In future, the denoising algorithms can be implemented on FPGA with reduced time complexity and power.

\section{References}

[1] P. Milanfar, "A tour of modern image filtering: new insights and methods, both practical and theoretical", IEEE Signal Processing and Magazines, Vol. 30, No. 1, pp. 106-128, 2013.

[2] C. Tomasi and R. Manduchi, "Bilateral filtering for gray and color images", In: Proc. of $6^{\text {th }}$ Int. Conf. on Computer Vision, Bombay, pp. 839846, 1998.

[3] A. Buades, B. Coll, and J. M. Morel, "A review of image denoising algorithms, with a new one", SIAM Journal on Multiscale Model and Simulation, Vol. 4, No. 2, pp. 490-530, 2005.

[4] K. He, J. Sun and X. Tang, "Guided image filtering", IEEE Transactions on Pattern Analysis and Machine Intelligence, Vol. 35, No. 6, pp. 1397-1409, 2013.

[5] D. L. Donoho and J. M. Johnstone, "Ideal spatial adaptation by wavelet shrinkage", Biometrika, Vol. 81, No. 3, pp. 425-455, 1994.

[6] D. L. Donoho, I. M. Johnstone, G. Kerkyacharian, and D. Picard, "Wavelet shrinkage: asymptopia", Journal of the Royal Statistical Society: Series B (Methodological), Vol. 57, No. 2, pp. 301-369, 1995.

[7] S. G. Chang, B. Yu, and M. Vetterli, "Adaptive wavelet thresholding for image denoising and compression", IEEE Transactions on Image Processing, Vol. 9, No. 9, pp. 1532-1546, 2000.

[8] L. Sendur and I. W. Selesnick, "Bivariate shrinkage with local variance estimation", IEEE Signal Processing Letters, Vol. 9, No. 12, pp. 438-441, 2002.

[9] A. Pizurica and W. Philips, "Estimating the probability of the presence of a signal of interest in multiresolution single- and multiband image denoising", IEEE Transactions on Image Processing, Vol. 15, No. 3, pp. 654-665, 206.
[10] T. Blu and F. Luisier, "The SURE-LET approach to image denoising", IEEE Transactions on Image Processing, Vol. 16, No. 11, pp. 2778-2786, 2007.

[11]Z. Dengwen and C. Wengang, "Image denoising with an optimal threshold and neighbouring window", Pattern Recognition Letters, Vol. 29, No. 11, pp. 1694-1697, 2008.

[12] J. L. Starck, E. J. Candès and D. L. Donoho, "The curvelet transform for image denoising", IEEE Transaction on Image Processing, Vol. 11, No. 6, pp. 670-684, 2002.

[13] M. Zhang and B. K. Gunturk, "Multiresolution bilateral filtering for image denoising", IEEE Transactions on Image Processing, Vol. 17, No. 12, pp. 2324-2333, 2008.

[14] C. Knaus and M. Zwicker, "Dual-domain image denoising", In: Proc. of 20th IEEE Int. Conf. on Image Processing, Melbourne, pp. 440-444, 2013.

[15] J. Ma and G. Plonka, "The curvelet transform", IEEE Signal Processing Magazine, Vol. 27, No. 2, pp. 118-133, 2010.

[16] K. Wu, X. Zhang, and M. Ding, "Curvelet based non-local means algorithm for image denoising", AEU International Journal on Electronics and Communication, Vol. 68, No. 1, pp. 37-43, 2014.

[17] S. Kumar, "Image denoising based on non-local means filter and its method noise thresholding", Signal, Image and Video Processing, Vol. 7, No. 6, pp. 1211-1227, 2013.

[18] V. G. Sivakumar, V. Rajendran, and R Saranya, "Comparison and analysis of vertical coherence in the shallow water of two ocean region", American Journal of Applied Sciences, Vol. 10, No. 6, pp. 542-548, 2013.

[19] K. Dabov, A. Foi, V. Katkovnik, and K. Egiazarian, "Image denoising by sparse 3-D transform-domain collaborative filtering", IEEE Transactions on Image Processing, Vol. 16, No. 8, pp. 2080-2095, 2007.

[20] C. Knaus and M. Zwicker, "Progressive image denoising", IEEE Transactions on Image Processing, Vol. 23, No. 7, pp. 3114-3125, 2014.

[21] Z. Zha, X. Liu, Z. Zhou, X. Huang, J. Shi, Z. Shang, L. Tang, Y. Bai, Q. Wang, and X. Zhang, "Image denoising via group sparsity residual constraint", In: Proc. of Int. Conf. on Acoustics, Speech and Signal Processing, pp. 1787-1791, 2017.

[22] A. Karami and L. Tafakori, "Image denoising using generalised Cauchy filter", IET Image Processing, Vol. 11, No. 9, pp. 767-776, 2017. 
[23] L. Lu, W. Jin, and X. Wang, "Non-local means image denoising with a soft threshold", IEEE Signal Processing Letters, Vol. 22, No. 7, pp. 833-837, 2015.

[24] X. Wang, W. Chen, J. Gao, and C. Wang, "Hybrid Denoise Method Based on Nonsubsampled Contourlet Transform and Bandelet Transform", IET Image Processing, Vol. 12, No. 5, pp. 778-784, 2018.

[25] S. K. Panigrahi, S. Gupta, and P. K. Sahu, "Curvelet-based multiscale denoising using non-local means \& guided image filter", IET Image Processing, Vol. 12, No. 6, pp. 909-918, 2018.

[26] M. N. Do and M. Vetterli, "The contourlet transform: an efficient directional multiresolution image representation", IEEE Transactions on Image Processing, Vol. 14, No. 12, pp. 2091-2106, 2005.

[27] E. J. Candes and D. Donoho, "Curvelets - a surprisingly effective nonadaptive representation for objects with edges", In: A. Cohen, C. Rabut and L. Schumaker, Editors, Curves and Surface Fitting: SaintMalo 1999, Vanderbilt University Press, Nashville, pp. 105-120.

[28] A. L. da Cunha, J. Zhou, and M. N. Do, "The Non-subsampled Contourlet Transform: Theory, Design and Applications", IEEE Transaction on Image Processing, Vol. 15, No. 10, pp. 3089-3100, 2006.

[29] Y. S. Kim, H. Lim, O. Choi, K. Lee, J. D. K. Kim, and C. Kim, "Separable bilateral nonlocal means," In: Proc. of IEEE International Conf. On Image Processing, pp. 1513-1516, 2011.

[30] C. Stein, "Estimation of the mean of a multivariate normal distribution", Annals of Statistics, Vol. 9, pp. 1135-1151, 1981.

[31] T. Blu and F. Luisier, "The SURE-LET approach to image denoising", IEEE Transactions on Image Processing, Vol. 16, No. 11, pp. 2778-2786, 2007.

[32] R. Deriche, "Recursively implementing the Gaussian and its derivatives", In: Proc. of IEEE International Conference on Image Processing, pp. 263-26, 1992. 